\title{
Pair Distribution Function from Electron Diffraction in Cryogenic Electron Microscopy: Revealing Glassy Water Structure
}

João Batista Souza Junior, ${ }^{1}$ Gabriel Ravanhani Schleder, ${ }^{1,2}$ Felippe Mariano Colombari, ${ }^{1}$ Marcelo Alexandre de Farias, ${ }^{1}$ Jefferson Bettini, ${ }^{1}$ Marin van Heel, $,{ }^{1}, 3,4$ Rodrigo Villares Portugal, ${ }^{1}$ Adalberto Fazzio, ${ }^{1,2}$ and Edson Roberto Leite ${ }^{1,5}$

${ }^{1}$ Brazilian Nanotechnology National Laboratory (LNNano), Brazilian Center for Research in Energy and Materials (CNPEM), 13083-970, Campinas, Brazil

${ }^{2}$ Federal University of ABC (UFABC), Santo André, São Paulo, Brazil

${ }^{3}$ Netherlands Centre for Electron Nanoscopy (NeCEN), 2333 CC Leiden, Netherlands

${ }^{4}$ Faculty of Natural Sciences, Imperial College London, London SW7 2AZ, UK

${ }^{5}$ Department of Chemistry, Federal University of

São Carlos, 13565-905, São Carlos, SP, Brazil

joao.junior@lnnano.cnpem.br; edson.leite@lnnano.cnpem.br 


\section{EXPERIMENTAL SECTION}

\section{A. Cryogenic Transmission Electron Microscopy (Cryo-TEM)}

TEM grids were subjected to an earlier glow discharge treatment using a Pelco easiGlow discharge system (Ted Pella, USA) for $25 \mathrm{~s}$ in air atmosphere, using a current of $15 \mathrm{~mA}$. A 3 $\mu \mathrm{L}$ deionized water droplet was then deposited on the 300 mesh holey carbon-coated copper grid (C-Flat 2/2, Electron Microscopy Sciences, USA). The samples were subsequently prepared in a controlled-environment vitrification system (Vitrobot Mark IV, Thermo Fischer Scientific, USA) at a temperature of $22{ }^{\circ} \mathrm{C}$ and $100 \%$ humidity. The samples were then transferred at liquid $\mathrm{N}_{2}$ temperature into a JEOL JEM-2100 electron microscope operating at $200 \mathrm{kV}$ and equipped with a CCD TVips camera $(4 \mathrm{k} \times 4 \mathrm{k}$ pixels $)$ to acquire the selected-area electron diffraction (SAED) with high $Q_{\max }\left(19.2 \AA^{-1}\right)$. To improve statistics at high $Q$ space, 10 frames were obtained using an integration time of $2000 \mathrm{~ms} /$ frame, a $30 \mathrm{~cm}$ camera length, and $40 \mu \mathrm{m} \mathrm{SA}$ aperture. We limited the total exposure dose to 20 $\mathrm{e}^{-} \AA^{-2} \mathrm{~s}^{-1}$ so as to stay within the typical dose levels used in cryo-EM. Each SAED image was submitted to a in-house image filter written in Python code to correct the values of the defective pixels. This was achieved by analyzing the mean and the standard deviation of the eight neighbouring pixels for each pixel on the image. If the analyzed pixel intensity was greater than 5 times the standard deviation of its neighbours, that pixel intensity was replaced to the minimum value among its neighbors. A second experiment, Figures S14-16, was done on TALOS F200C (Thermo Fischer Scientific, USA) electron microscope operating at $200 \mathrm{kV}$ to acquire the SAED for different known sample thicknesses. SAED images were acquired using a Ceta CMOS camera $(4 \mathrm{k} \times 4 \mathrm{k}$ pixels; by Thermo Fischer Scientific, USA) obtaining a total of 50 frames using an integration time of $300 \mathrm{~ms}$, a $850 \mathrm{~mm}$ camera length and $40 \mu \mathrm{m}$ SA aperture. The SAED patterns were acquired with a $Q_{\max }$ of $7.5 \AA^{-1}$. Sample thickness was estimated by tilting the sample at $45^{\circ}$, drilling a hole in the glassy ice with a highly focused beam, return to $0^{\circ}$ and measuring the length of the white trace within the hole, which will be equal to the thickness of the ice.

\section{B. Gatan Microscopy Suite (GMS 3) Data Preparation}

The Gatan Microscopy Suite (GMS 3) software version 3.31 was used to extract the scattering intensity profile $I(Q)$ from the SAED patterns. 
Center Location: first, the center of the diffraction pattern, the zero-order beam (000), was estimated using the DiffTools script. The results were compared interactively by matching the centers of the first three diffraction-rings. The mean center was used in DiffTools. The $\mathrm{x}-\mathrm{y}$ center values can be changed in the option "Image Display Info/Image/Tags/Diffraction Package/Center/X: and Y:".

Azimuthal Integration: the $I(Q)$ was obtained by rotational averaging the experimental CGW SAED image, excluding the area matching the beam stopper in the image. Using the script "Export Profile As Tabbed Text" from Dave Mitchell's Digital Micrograph ${ }^{\text {TM }}$ Scripting Website the $I(Q)$ data was saved as a txt file, which can directly be used in the the eRDF software [1].

Reciprocal space calibration: the scale of the $I(Q)$ was calibrated using a gold standard SAED pattern under the same TEM conditions (alignment, spot size, alpha, apertures, etc.). A first calibration SAED was obtained before the glassy water data collection and a second calibration SAED was recorded after the main CGW SAED experiment, Figure S2. The four first diffraction peaks positions $\left(I_{h k l}^{\max }\right)$ related to the (111), (200), (220), and the (311) interplanar spacing $d_{h k l}$ were used to calibrate the reciprocal space (ds) as $\AA^{-1} /$ pixel, being $d s=1 / d_{h k l} * I_{h k l}^{\max }$. The mean value between the two calibration SAED experiments were used for this purpose. This calibration was also used in eRDF to lead to the vector $s$ scale $(s=Q / 2 \pi=1 / d)$ that needs to be used as input file to finally compute the $Q$ space as $Q=(4 \pi \sin \theta) / \lambda=2 \pi / d=2 \pi s\left(\AA^{-1}\right)$.

\section{C. eRDF Analysis}

The calibrated $I(Q)$ file obtained previously was used as input file for eRDF. The matlab code was modified to obtain the $g(r)$ for CGW as follows. Following Skinner et al. [2] and Mariedahl et al. [3], in order to obtain the molecular form factor $\mathrm{S}(\mathrm{Q})_{\text {mol }}$, instead of using the atomic form factor $\mathrm{S}(\mathrm{Q})_{a t}$ that contains both intra- and intermolecular scattering, a series of data treatment was performed. First, the electron atomic form factor $f_{e, x}^{0}$ obtained by the Kirkland equations implemented in eRDF was corrected to account for the O-H bond formation on water that changes the intramolecular electron density and consequently the atomic form factor. The Sorenson et al. equation was used [4], generating both the modified atomic form factor $\left(\mathrm{MAFF}_{e}\right)$, the $f_{e, x}$ for oxygen $f_{e, O}$, and the $f_{e, H}$ for hydrogen, Equation 
(1):

$$
f_{e, x}(Q)=f_{e, x}^{0}(Q)\left[1-\frac{a_{x}}{z_{x}} \exp \left(-Q^{2} / 2 \delta^{2}\right)\right]
$$

being $z_{x}$ the atomic number of the element $x(\mathrm{O}$ or $\mathrm{H}), a_{O}=-1$ and $a_{H}=0.5$. Then, the electron molecular form factor C(Q) was calculated using the Equation (2).

$$
C(Q) \cong f_{e, O}^{2}+2 f_{e, H}^{2}+4 f_{e, O} f_{e, H}+4 f_{e, H}^{2}
$$

Using $\mathrm{C}(\mathrm{Q})$ as the weighing form factor, the molecular structure factor $\mathrm{S}(\mathrm{Q})_{\text {mol }}$ was obtained using Equation (3).

$$
S(Q)_{m o l}-1=\frac{I(Q)-C(Q)}{C(Q)}
$$

However, on eRDF the scaling of $\mathrm{I}(\mathrm{Q})$ (in electrons count units, depending on data acquisition) with $\mathrm{C}(\mathrm{Q})$ was done iteratively by using an Auto fit function to fulfill the PDF criteria that $\mathrm{S}(\mathrm{Q})$ must approach unity for $Q \rightarrow \infty$ :

$$
S(Q)_{a v g}=\lim _{Q \rightarrow \infty}\left\langle S(Q)_{m o l}\right\rangle=1
$$

The Auto fit was performed on the tail region of the experimental data $I(Q)$ to satisfy Equation (4). A scale factor $N$ on the numerator $(I(Q)-N \times C(Q))$ leads to Equation (5).

$$
S(Q)_{m o l}-1=\frac{I(Q)-\text { Autofit }}{\text { Autofit }}
$$

being Autofit $=N \times C(Q)$. Then, using the manual fit, the fitting was interactively tuned by changing the $N$ value to satisfy a second PDF property:

$$
S(Q)_{i n t}=\int_{0}^{\infty} Q^{2}[S(Q)-1] d Q=-2 \pi^{2} \rho_{0}=-0.608
$$

Once the $S(Q)_{m o l}$ was obtained, the contribution of intermolecular O-H and $\mathrm{H}-\mathrm{H}$ could then be subtracted from the $\mathrm{S}(\mathrm{Q})_{\text {mol }}$ to obtain the $S_{O O}(Q)$ that has only the structure information from the oxygens, i.e. the molecular structure. Equation (7) was used, being $\omega_{O O}=\frac{f_{e, O}^{2}}{9 C(Q)}$, $\omega_{O H}=\frac{4 f_{e, O} f_{e, H}}{9 C(Q)}$, and $\omega_{H H}=\frac{4 f_{e, H}^{2}}{9 C(Q)}$.

$$
S_{O O}(Q)=\frac{S(Q)_{m o l}-\omega_{H H} \times 1-\omega_{O H} \times S_{O H}(Q)}{\omega_{O O}}
$$


Where the $S_{O H}(Q)$, Figure SS3, was calculated from the $g_{O H}(r)$ obtained from our molecular dynamics results for supercooled water as follow:

$$
\begin{gathered}
G_{O H}(r)=4 \pi \rho_{0} r \gamma(r)\left(g_{O H}(r)-1\right) \\
Q\left[S_{O H}(Q)-1\right]=\int_{0 \AA}^{150 \AA} G_{O H}(r) \sin (Q r) d r
\end{gathered}
$$

Similarly, the $S_{O O}(Q)$ was calculated from the $g_{O O}(r)$ to compare with the final experimental $S_{O O}(Q)$, Figure SS5. The $S_{O H}(Q)$ data was compared to $S_{O H}(Q)$ from Zeidler et al. obtained from oxygen isotope-substituted neutron scattering data for liquid water, see Figure SS3 $[5,6]$. Both results were tested to subtract the $\mathrm{OH}$ and to obtain our experimental $S_{O O}(Q)$, however, the $S_{O H}(Q)$ from molecular dynamics lead to a better $\mathrm{OH}$ peak removal at $1.0 \AA$, therefore being used in our final results. Experimental $G(r)$ and $g(r)$ were then obtained by Fourier transforming the reduced structure function $F(Q)=Q[S(Q)-1]$, using Equation (10):

$$
G_{O O}(r)=\frac{2}{\pi} \int_{0}^{\infty} Q[S(Q)-1] \times \sin (Q r) d Q
$$

A band-pass filter was also added to eRDF following Ref. [7] (SuePDF software), where the low $r$ region at distances smaller than the first O-O peak in the $G_{O O}(r)$ was replaced by:

$$
G_{O O}(r)_{0 \AA}^{2.4 \AA}=-4 \pi \rho_{0} r \gamma(r)
$$

and the high-frequency noise was eliminated by filtering out the oscillations beyond $15 \AA$ by letting the $G_{O O}(r)=0$, Figure SS7. The average number density for CGW, $\rho=0.0308$ $\AA^{-3}$, was estimated by using the unit cell volume (CIF file ICSD 64776) and considering the density between the LDA phase $\left(0.94 \mathrm{~g} \mathrm{~cm}^{-3}\right)$ and crystalline ice $\left(0.917 \mathrm{~g} \mathrm{~cm}^{-3}\right)$. The final $S_{O O}(Q)^{*}, G_{O O}(r)^{*}$, and $g_{O O}(r)$ are calculated as follows:

$$
\begin{gathered}
Q\left[S_{O O}(Q)^{*}-1\right]=\int_{0 \AA}^{150 \AA} G_{O O}(r) \sin (Q r) d r \\
G_{O O}(r)^{*}=\frac{2}{\pi} \int_{0 \AA-1}^{150 \AA^{-1}} M(Q, \Delta(r)) Q\left[S(Q)^{*}-1\right] \times \sin (Q r) d Q \\
g_{O O}(r)=1+\frac{1}{2 \pi^{2} \rho r} \int_{0 \AA-1}^{150 \AA^{-1}} M(Q, \Delta(r)) Q\left[S(Q)^{*}-1\right] \times \sin (Q r) d Q
\end{gathered}
$$


where $\mathrm{M}(\mathrm{Q}, \Delta(r))$ is a $r$-dependent Lorch-like function that is used to suppress the nonphysical truncation oscillations that comes from the finite $Q_{\max }\left(19.28 \AA^{-1}\right)$ :

$$
\begin{gathered}
M(Q, \Delta(r))=\frac{\sin (Q \Delta(r)}{Q \Delta(r)} \\
\Delta(r)=\frac{\pi}{Q_{\max }}\left[1-\exp \left(\frac{-\left|r-r_{1}\right|}{\omega_{1}}\right)\right]+a\left(\frac{1}{2}+\frac{1}{\pi} \arctan \left(\frac{r-\omega_{2}}{\omega_{2} / 2 \pi}\right)\right) \sqrt{r}
\end{gathered}
$$

being $r_{1}$ the center position of the first O-O peak, $2.77 \AA, \omega_{1}$ the width parameter of $0.3 \AA$, and $\omega_{2}=12 \AA$. The procedure is described in Refs. [2] and [3], see Figure SS7. Finally, the O-O coordination number $n_{O O}(r)$ was calculated to determine the number of atoms as a function of distance $r$ by integrating the PDF $g_{O O}(r)$ :

$$
n_{O O}(r)=4 \pi \rho \int_{0}^{r} r^{2} g_{O O}(r) d r
$$

where $\rho$ is the number density for the specific sample.

\section{COMPUTATIONAL DETAILS}

\section{A. Pair Distribution Function}

The pair distribution function $g(r)$ simulated for both hexagonal $\left(\mathrm{I}_{h}\right.$, P63mmc space group, ICSD 64776) and cubic ( $\mathrm{I}_{c}, \mathrm{Fd} 3-\mathrm{m}$ space group, ICSD 29066) crystalline ice (bulk) were generated from the crystal structure CIF files using the OVITO analysis software [8], accounting for the selected atomic pairs (O-O). We used a resolution of $0.01 \AA$. To compare the PDFs calculated with experiments, we applied a one-dimensional gaussian convolution filter with a $\sigma=10$ value.

\section{B. Molecular Dynamics Simulations}

Classical molecular-dynamics simulations were performed for liquid water, supercooled water and for low density amorphous ice (LDA) using GROMACS 2019.1 package [9, 10]. First, an initial liquid system was built by placing 6144 TIP4P/ice [11] molecules randomly

inside a $58 \AA$ x $58 \AA$ x $58 \AA$ box. Repulsive molecular contacts were removed by performing an energy minimization with the steepest-descent algorithm until all forces reached values below $5 \mathrm{~kJ} / \mathrm{mol} / \AA$. Trajectories for all aforementioned systems were then generated at 
the isobaric-isothermic (NpT) ensemble, considering different thermalization and annealing schemes:

\section{- Liquid water}

A 110 ns long simulation was performed with $\mathrm{T}=298.15 \mathrm{~K}$ and $\mathrm{p}=1$ bar. Initial 50 ns were discarded prior to thermalization and density equilibration. Final 60 ns were considered for further analysis.

- Liquid water $\rightarrow$ Hyperquenched glassy water $(H G W) \rightarrow$ Low density amorphous ice $(L D A)$

The resulting liquid-water structure was then cooled down to $77 \mathrm{~K}$ using the following annealing scheme at $\mathrm{p}=1$ bar:

i) $0 \mathrm{~ns}-5 \mathrm{~ns}: 298.15 \mathrm{~K}$;

ii) $5 \mathrm{~ns}-55 \mathrm{~ns}: 298.15 \mathrm{~K}$ to $77 \mathrm{~K}$;

iii) $55 \mathrm{~ns}$ to $60 \mathrm{~ns}$ : equilibration of HGW structure at $77 \mathrm{~K}$;

iv) $60 \mathrm{~ns}$ to $110 \mathrm{~ns}$ : sampling of HGW structure.

The LDA ice structure was then obtained by heating HGW structure at $\mathrm{p}=1$ bar using the annealing:

v) $0 \mathrm{~ns}-5 \mathrm{~ns}: 77 \mathrm{~K}$;

vi) $5 \mathrm{~ns}-55 \mathrm{~ns}: 77 \mathrm{~K}$ to $130 \mathrm{~K}$;

vii) 55 ns to 60 ns: equilibration of LDA structure at $130 \mathrm{~K}$;

viii) $60 \mathrm{~ns}$ to $110 \mathrm{~ns}$ : data collection of LDA structure.

\section{- Liquid water $\rightarrow$ supercooled water}

Similarly, the supercooled water structure was sampled by cooling the liquid water structure down to $233 \mathrm{~K}$ at $\mathrm{p}=1$ bar:

i) $0 \mathrm{~ns}-5 \mathrm{~ns}: 298.15 \mathrm{~K}$;

ii) $5 \mathrm{~ns}-55 \mathrm{~ns}: 298.15 \mathrm{~K}$ to $233 \mathrm{~K}$;

iii) $55 \mathrm{~ns}$ to $60 \mathrm{~ns}$ : equilibration of supercooled water structure at $233 \mathrm{~K}$;

iv) $60 \mathrm{~ns}$ to $110 \mathrm{~ns}$ : sampling of supercooled water structure. 
Temperature and pressure were controlled using Berendsen weak-coupling schemes [12]. Newton equations of motion were integrated using a 2 fs timestep. All calculations were performed using standard 3D periodic-boundary conditions and electrostatic interactions were treated by means of a particle-mesh Ewald summation with a cutoff of $12 \AA[13$, 14]. Non-bonded interactions were calculated up to $10 \AA$, and a switching function was used to bring them to zero at $12 \AA$. Analytic corrections to pressure and potential energies were used to compensate for the truncation of the Lennard-Jones interactions [15]. The rigid geometry of TIP4P/ice molecules was enforced with the SETTLE algorithm [16] while additional constraints were treated via the P-LINCS algorithm [17]. Radial distribution functions were calculated for O-O pair correlations using a bin-width of $0.01 \AA$. The spatial distribution functions (SDFs) were obtained by evaluating 21 equally-spaced frames of the last 210 MD steps of each trajectory with the TRAVIS [18] software package, using a secondorder smoothing function. 


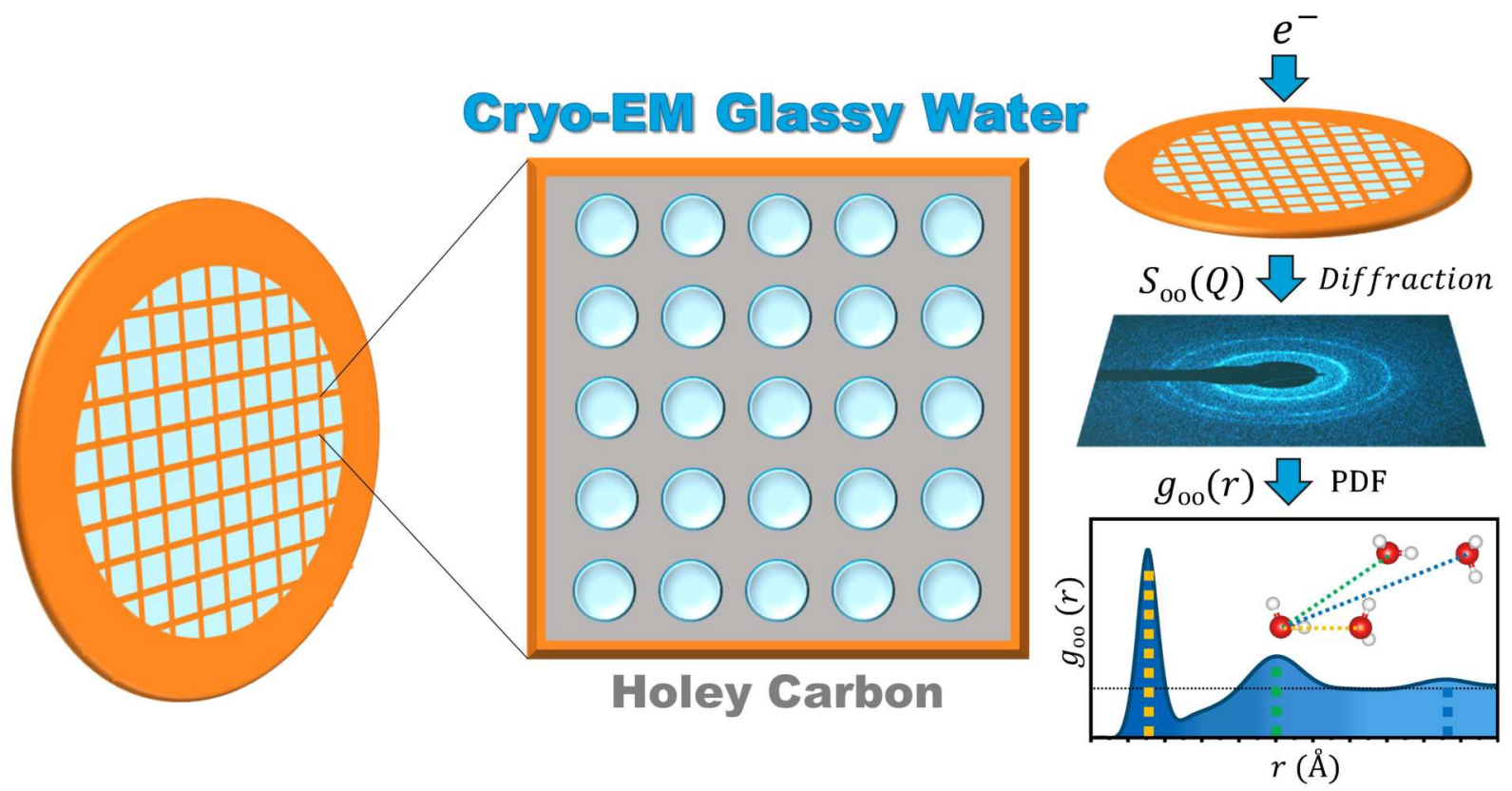

FIG. S1. Scheme of Cryo-EM TEM grid used as sample holder for vitreous ice experiments illustrating the holey carbon-coated copper grid with holes filled with ice of different thicknesses. The electron diffraction pattern is collected and used for extracting the structure function $S_{O O}(Q)$ and, from that, the pair distribution function $\operatorname{PDF} g_{O O}(r)$ is calculated as the Fourier transform of $S_{O O}(Q)$. 

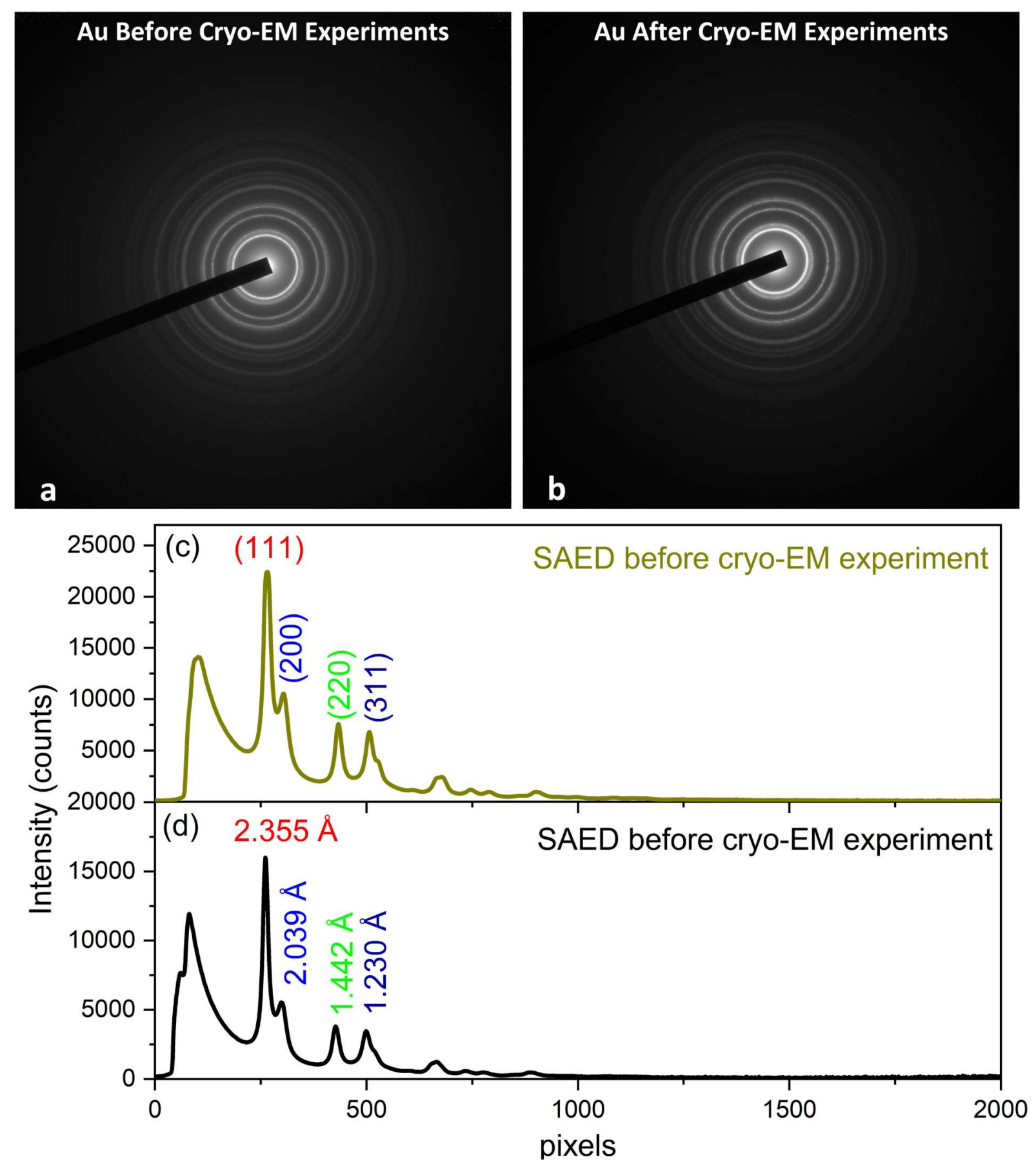

FIG. S2. Electron diffraction patterns (SAED) for gold samples acquired before (a) and after (b) the Cryo-EM glassy-water SAED experiments, for the calibration of reciprocal space (ds, $\AA^{-1} /$ pixels). Less than $\sim 1 \%$ change in camera length was observed for the (111) peak. Intensity profiles (c) and (d) for diffraction patterns (a) and (b), respectively. 


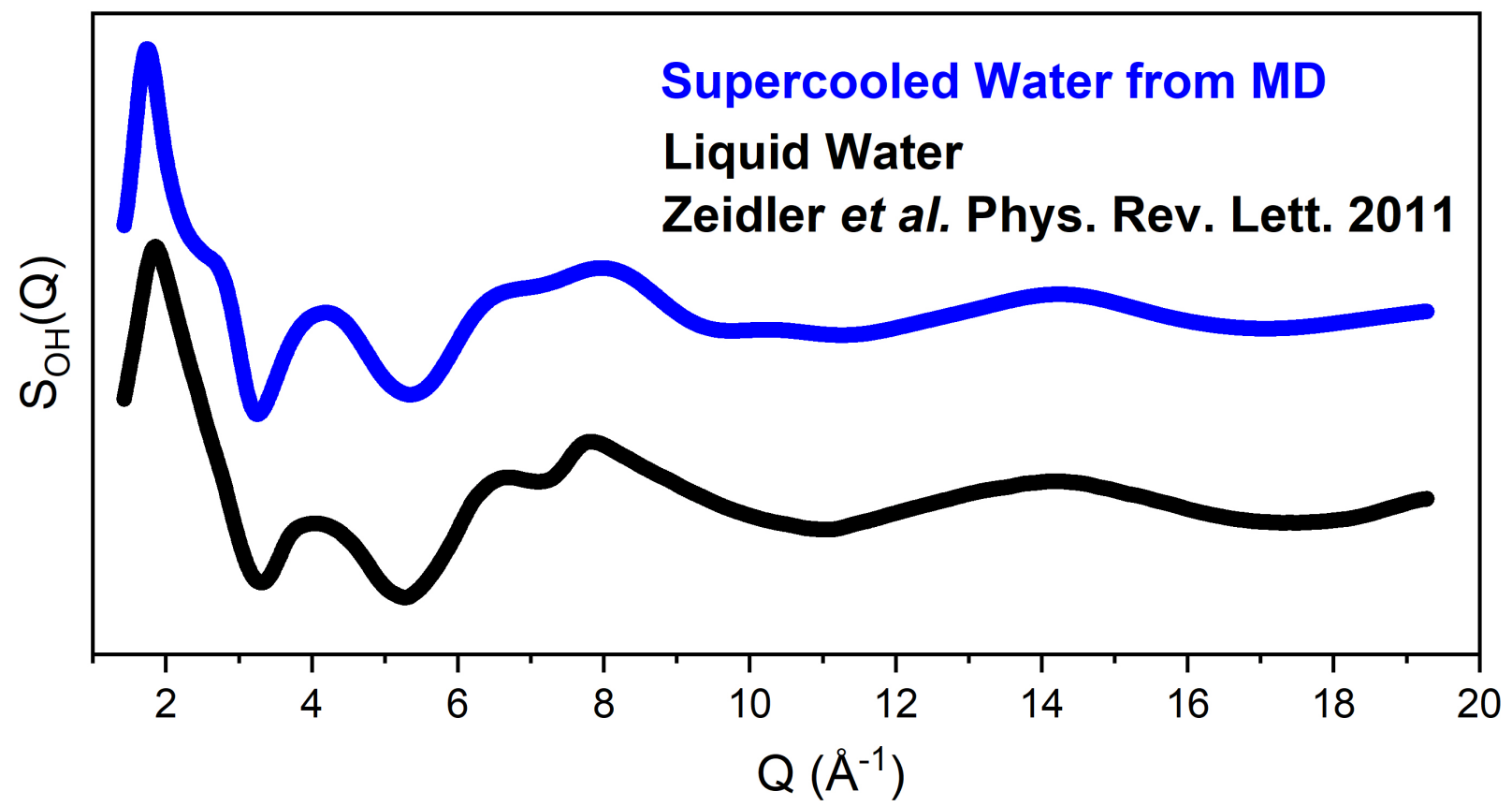

FIG. S3. $S_{O H}(Q)$ calculated from the $G_{O H}(r)$, Equation S9, for the supercooled water obtained from the molecular dynamics compared to Zeidler et al. experimental results obtained from Neutron diffraction for liquid water $[5,6]$. The calculated $S_{O H}(Q)$ was used to subtract the intermolecular O-H peak. 


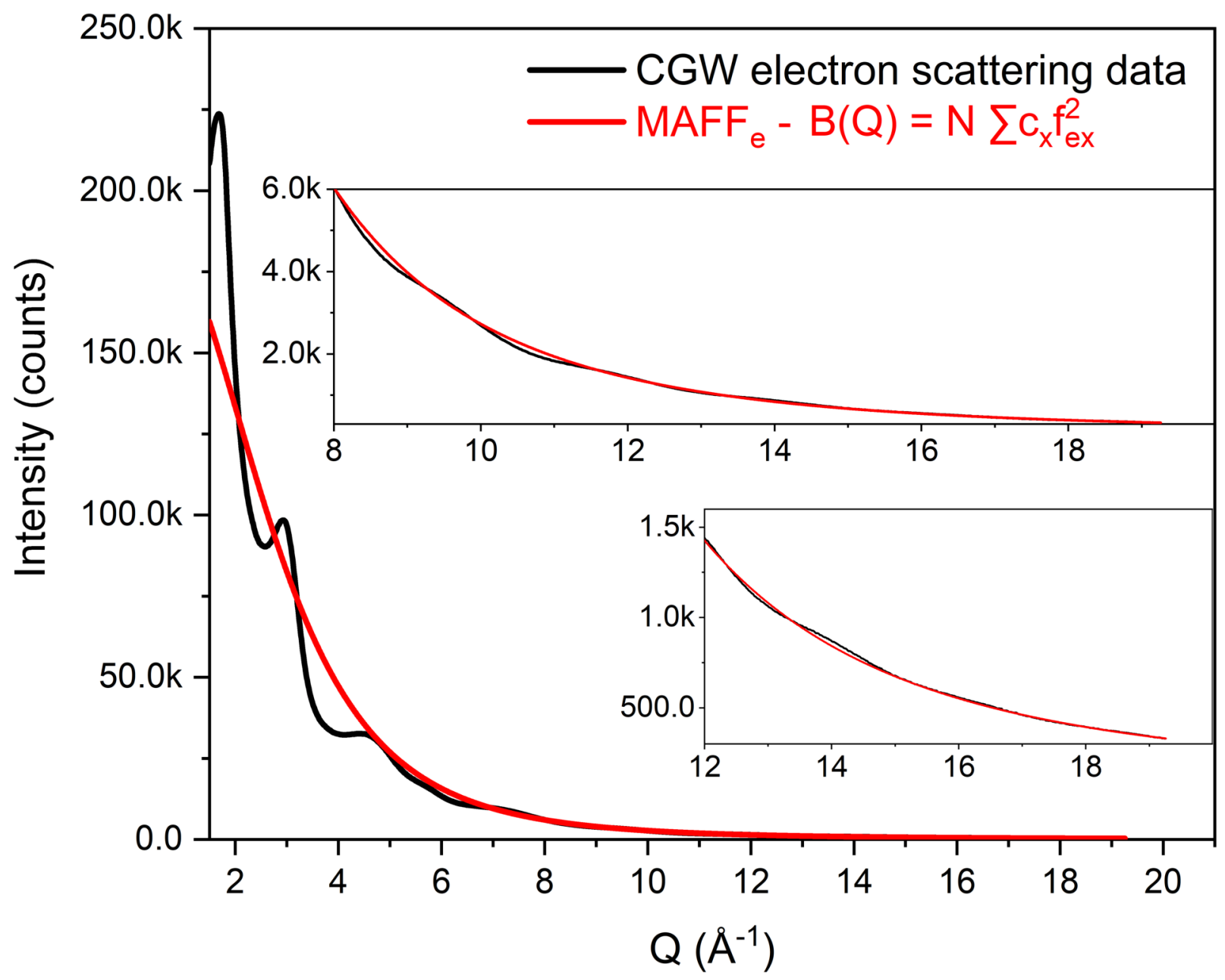

FIG. S4. Electron scattering data $I(Q)$ obtained by the sum of ten electron diffraction images (azimuthal integration) along with the modified atomic form factor $\left(\mathrm{MAFF}_{e}\right)$ used to calculate the molecular form factor $C(Q)$ by using the $\mathrm{MAFF}_{e}$ for oxygen and hydrogen. 


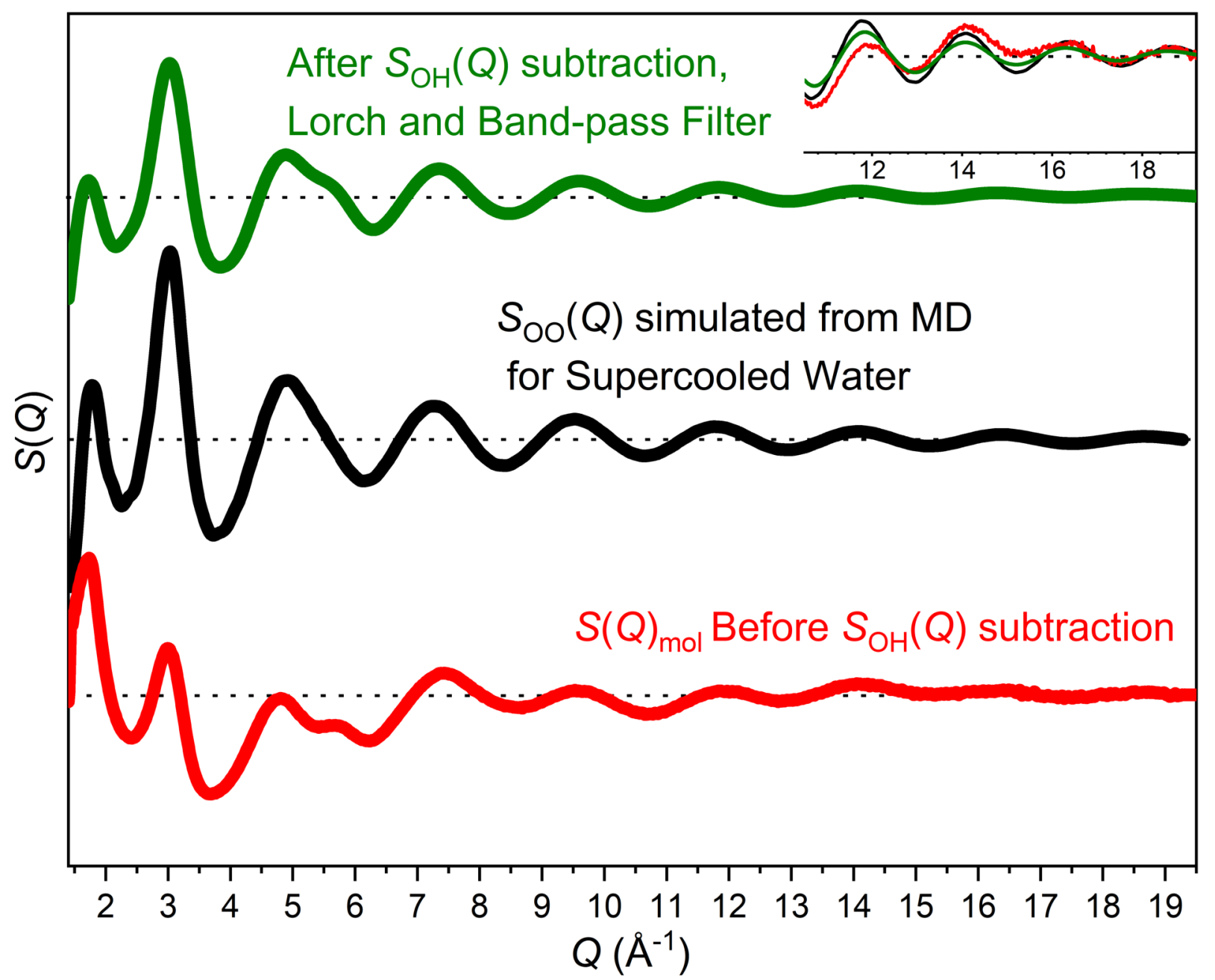

FIG. S5. Structure factor $S(Q)$ for the CGW sample before $\left(S(Q)_{m o l}\right.$, red curve) the subtraction of the $S_{O H}(Q)$ and the final result obtained after the $S_{O H}(Q)$ subtraction and after using the Band-Pass $(2.4-15 \AA)$ and Lorch filter $\left(S_{O O}(Q)\right.$, green curve). The $S_{O O}(Q)$ from the supercooled water from the MD is displayed for comparison (black curve). 


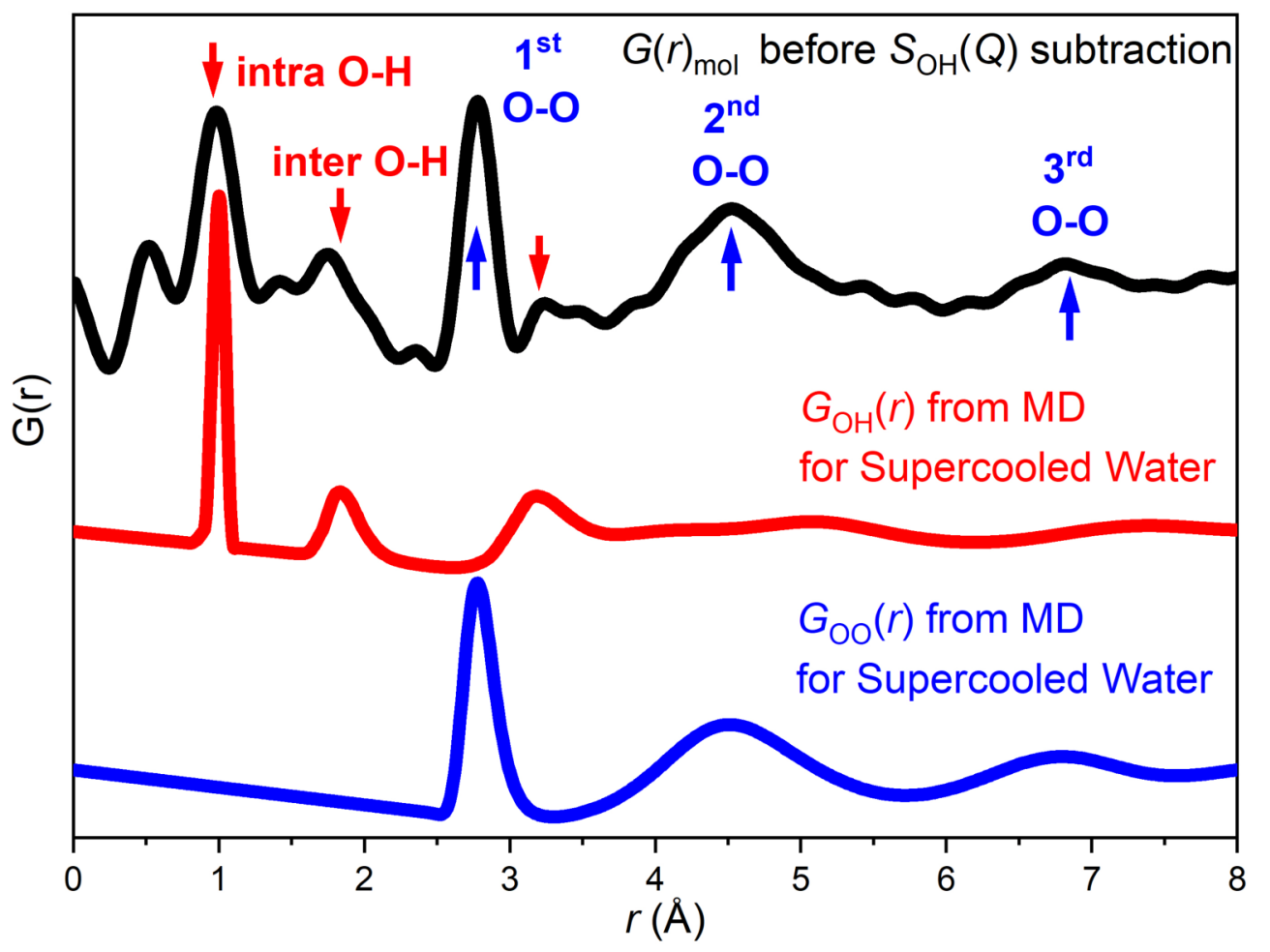

FIG. S6. Molecular reduced PDF $G(r)_{\text {mol }}$ obtained before any data treatment showing the presence of O-O peaks and O-H peaks. The $G_{O O}(r)$ and the $G_{O H}(r)$ from the MD from the supercooled water is also displayed to compare the peaks with the $G(r)_{m o l}$ experimental data. 


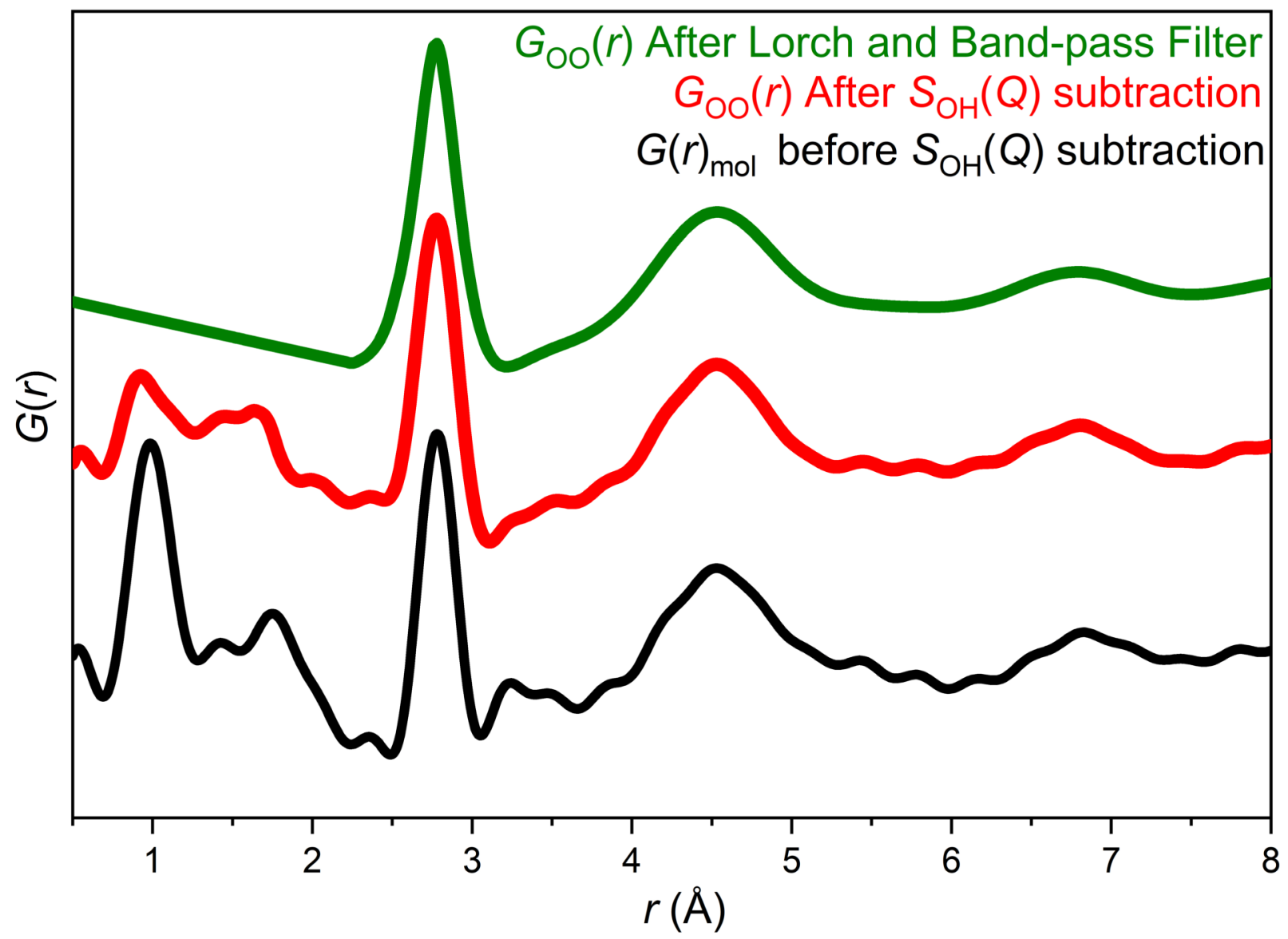

FIG. S7. Molecular reduced PDF $G(r)_{m o l}$ before (black) and after $\left(G_{O O}(r)\right.$, red) the $S_{O H}(Q)$ subtraction, being the final result (green) also submitted to a band-Pass filter (2.4-15 $\AA$ ) and a Lorch filter. 


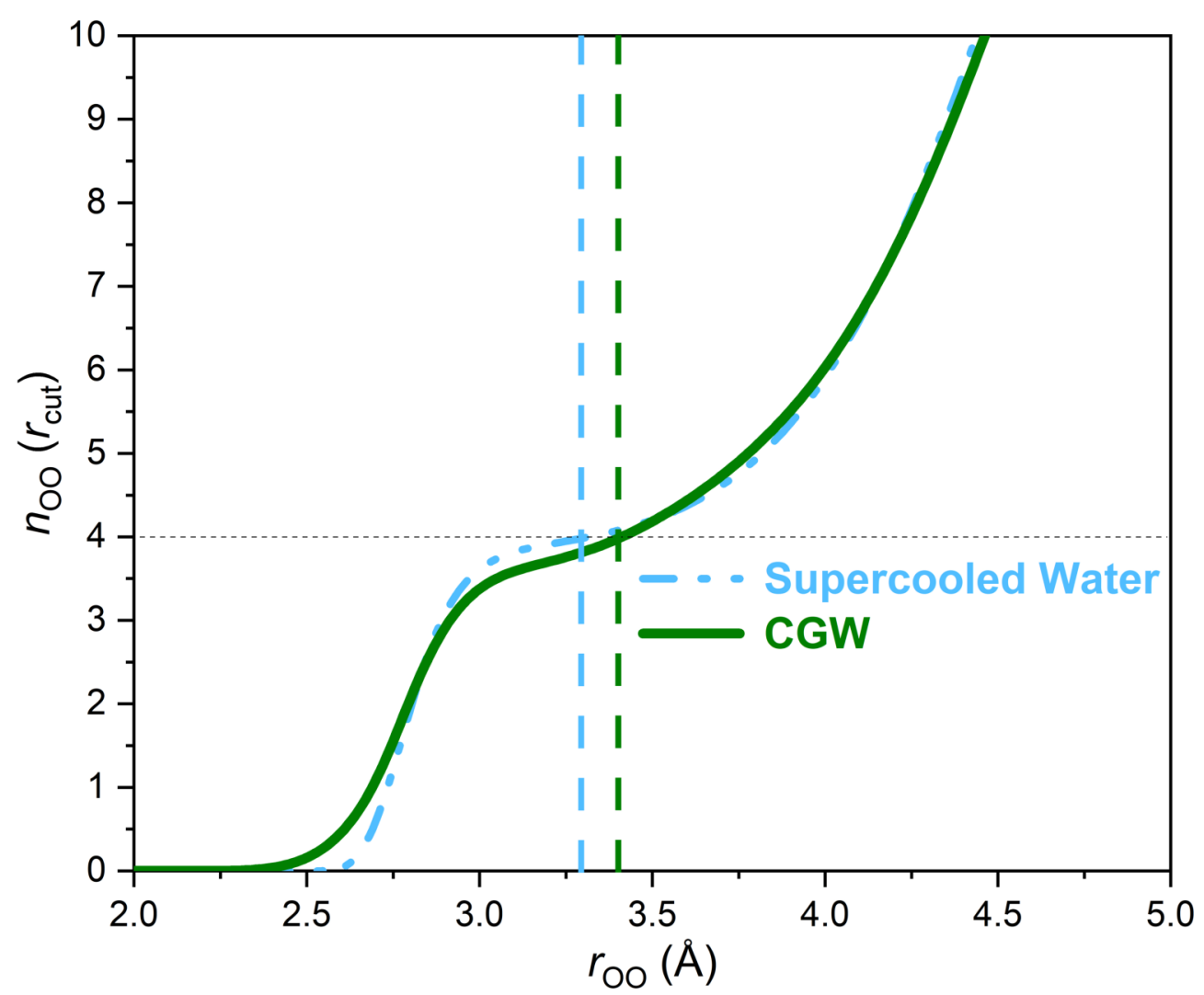

FIG. S8. O-O coordination number $n_{O O}$ for the CGW (green curve) and the supercooled water (blue curve) showing the location of $n_{O O}=4$ 

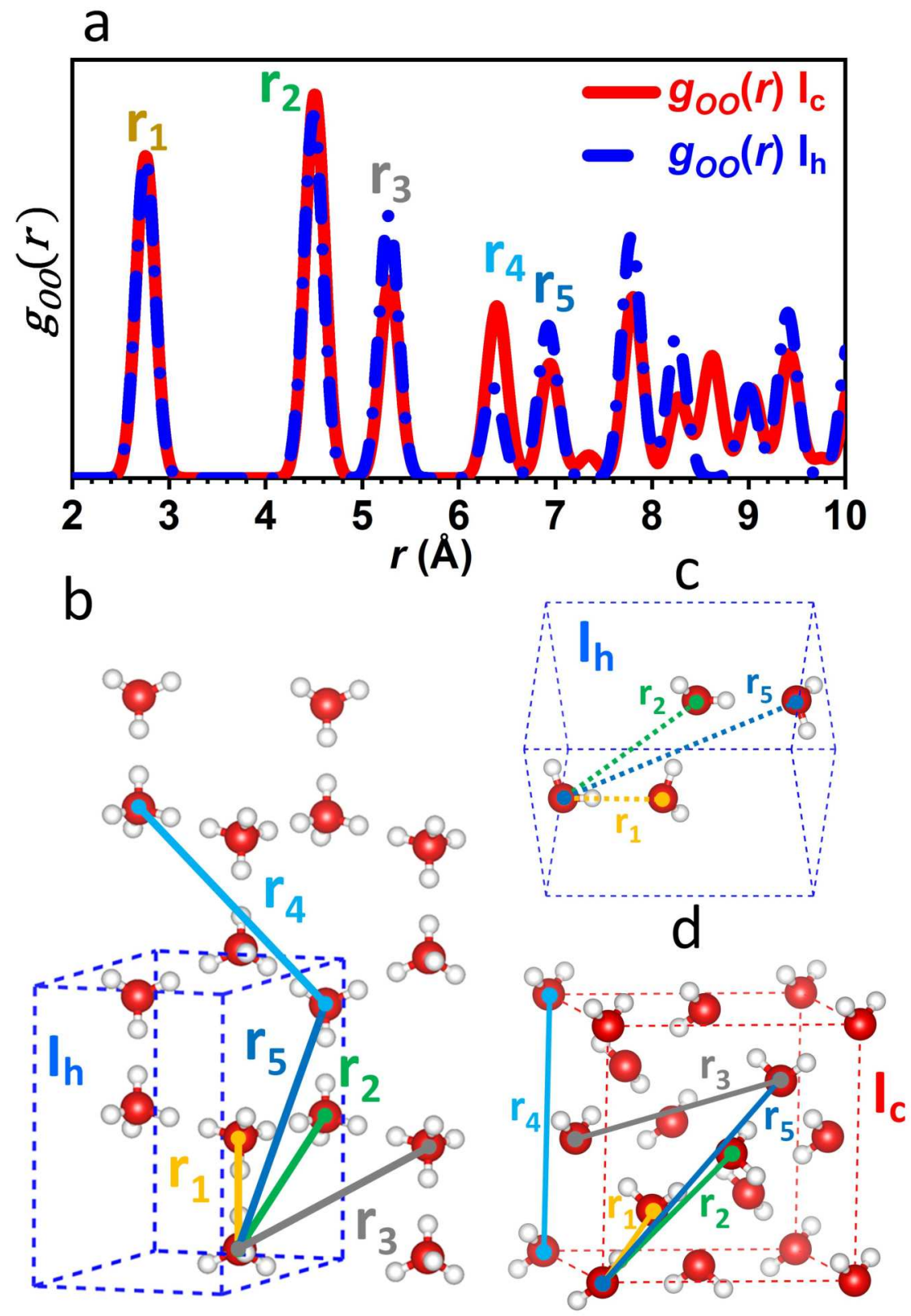

FIG. S9. (a) Simulated O-O PDF $g_{O O}(r)(r)$ for both hexagonal $\left(\mathrm{I}_{h}\right)$ and cubic $\left(\mathrm{I}_{c}\right)$ bulk crystalline ice showing the O-O distances ( $r$ coloured vectors) that can be visualized in both (b-c) $\mathrm{I}_{h}$ and (d) $\mathrm{I}_{c}$ unit cells. 

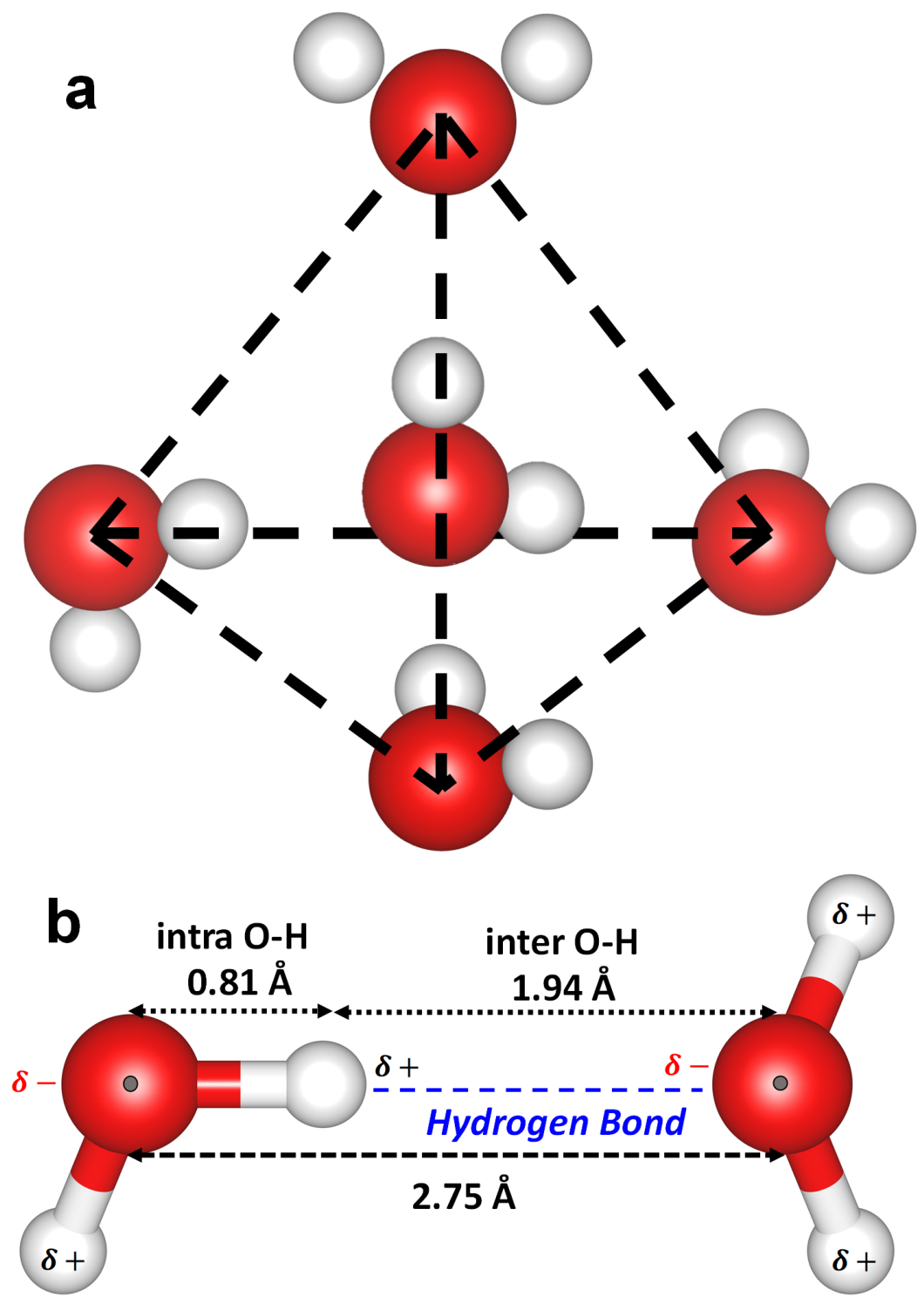

FIG. S10. (a) Water tetrahedra generated by the $\mathrm{I}_{h}$ structure (hexagonal system, $P 6{ }_{3} m m c$ space group, ICSD 64776) using VESTA ${ }^{\circledR}$ Software showing that the central $\mathrm{H}_{2} \mathrm{O}$ molecule have four hydrogen bonds, two intra-molecular $\mathrm{O}-\mathrm{H}$ bond to two other oxygen and two $\mathrm{O}-\mathrm{H}$ through the lone pairs. The same structure can be obtained using the $\mathrm{I}_{c}$ structure (cubic, $F d 3 \bar{m}$ space group, ICSD 29066), which is correlated with the $g_{1}(2.75 \AA)$, the first coordination shell. (b) Distance of the hydrogen bond and $\mathrm{O}-\mathrm{O}$ distance between neighboring water molecules. 


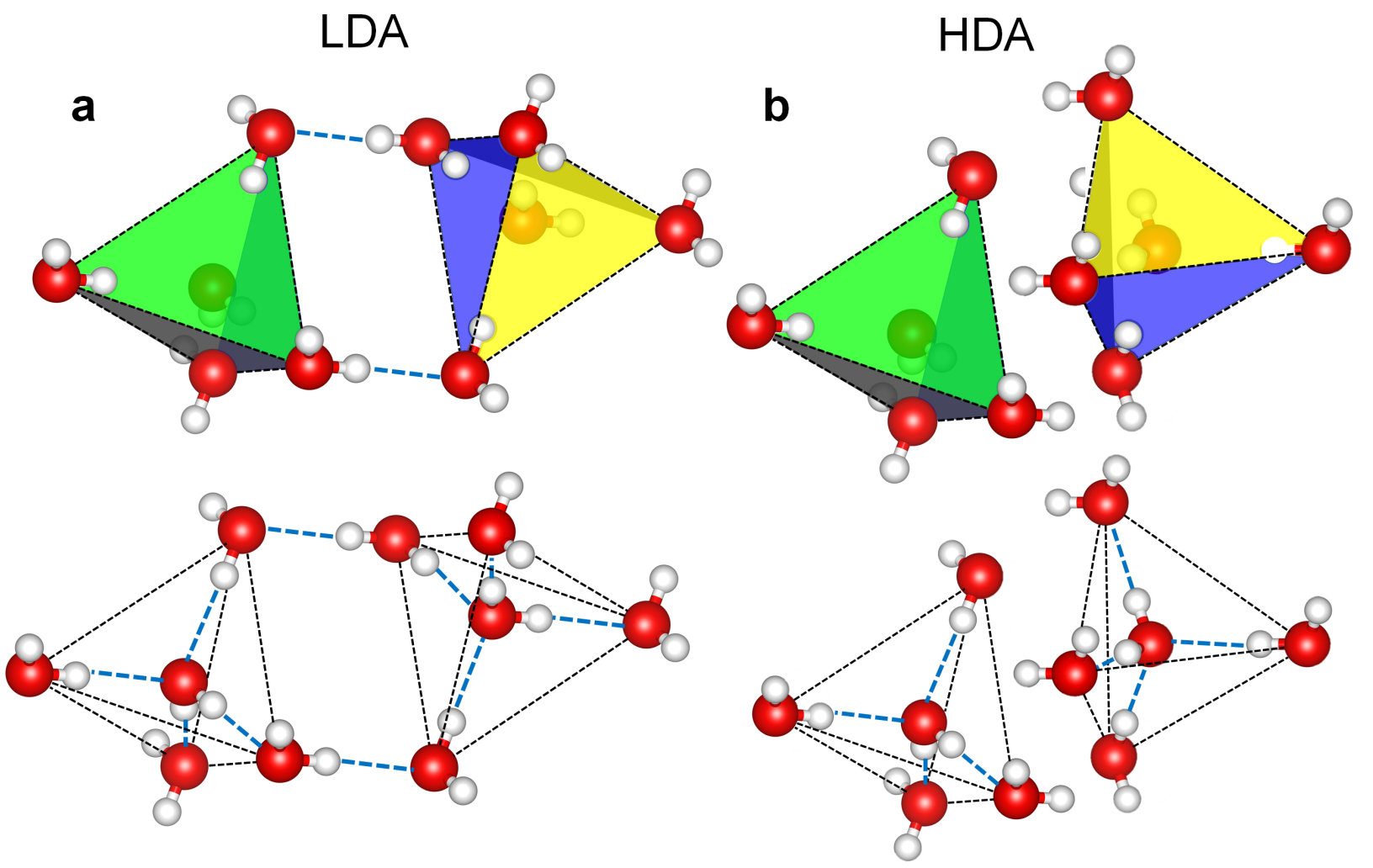

FIG. S11. (a) Depicting a low-density amorphous ice (LDA ice) with a double hydrogen-bond interaction between two tetrahedra. The local LDA structure presents two nearest neighbor tetrahedra connected by these two hydrogen bonding interactions, but not keeping the long-range ordering. If a long-range ordering is kept, i.e. all $\mathrm{H}_{2} \mathrm{O}$ molecules possessing a symmetrically connected tetrahedra through hydrogen bonding (dotted blue lines), a crystalline ice $\left(\mathrm{I}_{c}\right.$ or $\left.\mathrm{I}_{h}\right)$ would be formed. In fact, both local structure of $\mathrm{I}_{h}$ and $\mathrm{I}_{c}$ ice have this LDA structure (two connected tetrahedra). (b) On the other hand, the high density amorphous (HDA) structure do not keep the local structure between two nearest neighbor tetrahedra and the two oriented hydrogen bonds are lost. Consequently, one or more $\mathrm{H}_{2} \mathrm{O}$ molecules from one tetrahedra can be located closer to another tetrahedra. 


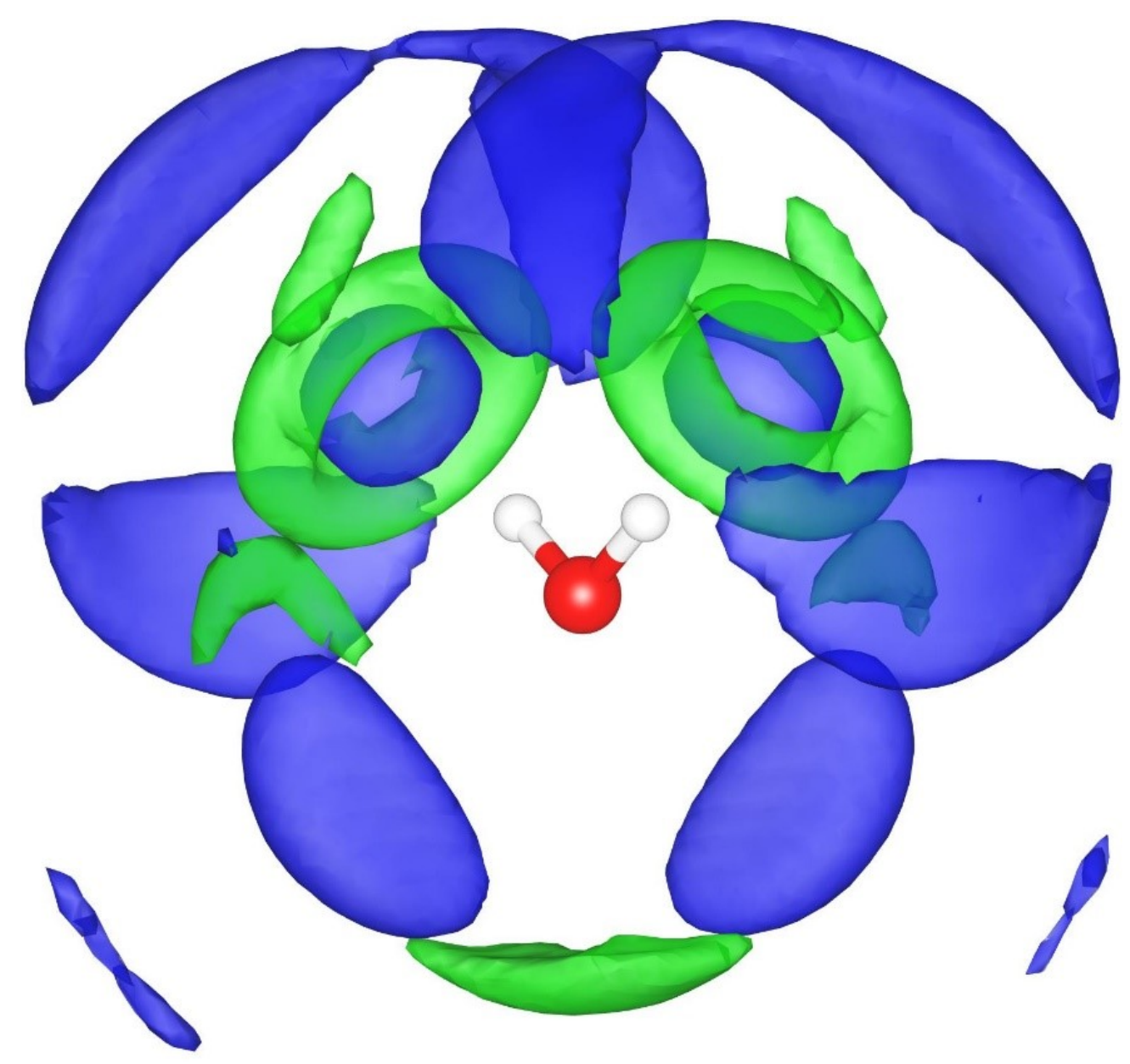

FIG. S12. Spatial distribution function (SDF) difference between liquid water and LDA, calculated using molecular dynamics results. Isosurface value of 35 molecules $\mathrm{nm}^{-3}$; green and blue regions denote positive and negative values, respectively. Positive values (green) indicate regions with larger spatial concentration of molecules for liquid water in relation to LDA, while negative values (blue) indicate the opposite. Liquid water presents orientational deviations (radial and angular deviations from ideal tetrahedral network) and also correlations in the depletion region, related to its dynamical behaviour (green regions). Contrarily, LDA shows a greater degree of regularity in comparison with liquid water (blue regions), presenting a more ordered tetrahedral network structure beyond first neighbours (which are the two blue lobes in the direction of oxygen lone pairs, and the two blue spheres surrounded by the green toroids). 


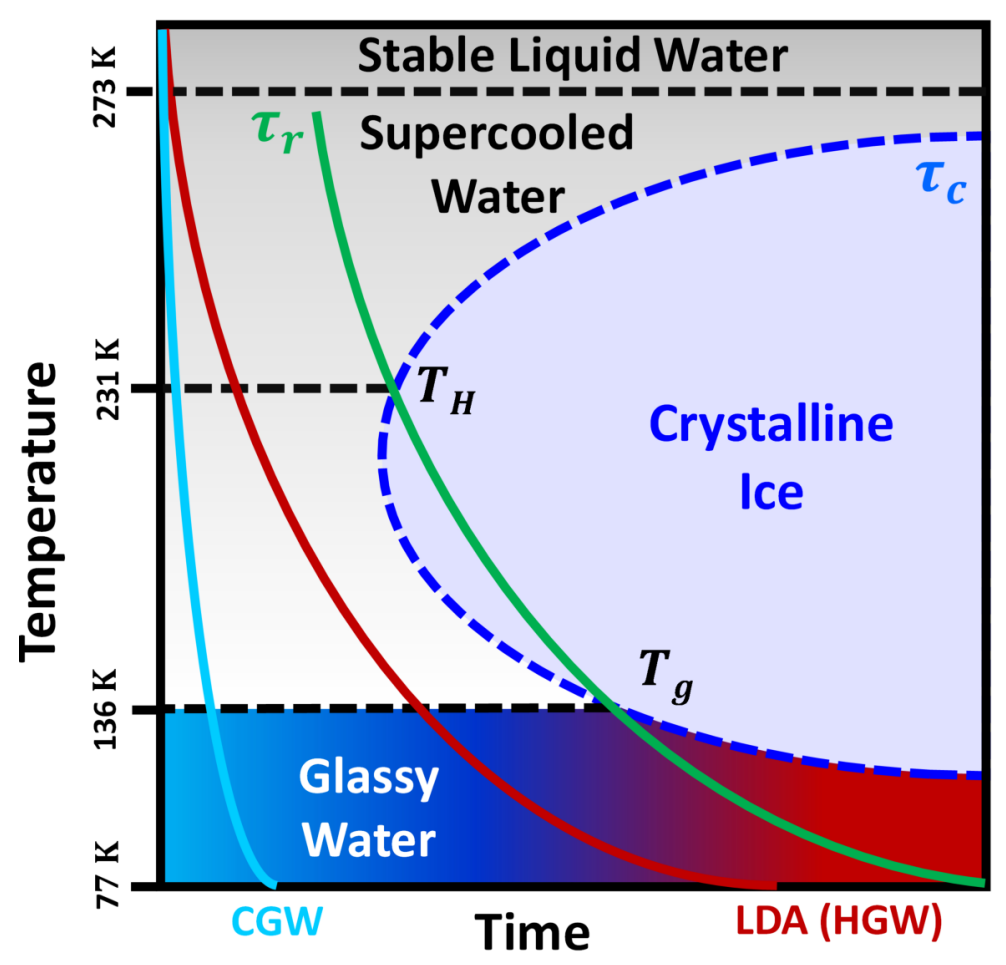

FIG. S13. Hypothetical time-temperature diagram for water cooling. It is shown the water crystallization time $\tau_{c}$ and water's structural relaxation time $\tau_{r}$. The $\tau_{r}$ line intercepts the $\tau_{c}$ two times, one nearby the glass transition temperature $\mathrm{T}_{g}$ and another nearby the homogeneous nucleation temperature $\mathrm{T}_{H}(231 \mathrm{~K}$, which is also close to the Widow line transition from HDL to LDL at ambient pressure, 229.2 K) [19, 20]. We present two scenarios for amorphous ice formation: first, a cooling rate that leads to Cryo-EM glassy water (CGW), blue curve, and a second that leads to hyperquenched glassy water (HGW, similar to LDA structure), red curve; both cooling rates are faster than water crystallization time. The bottom of the graphic around $77 \mathrm{~K}$ indicates the liquid nitrogen temperature, the final temperature for CGW experiments. Although the CGW was initially produced at $90 \mathrm{~K}$ (ethane melting point), all cryo-EM experiments were conducted in liquid nitrogen. The ethane used for cooling in cryo-EM has a higher heat capacity $\left(\mathrm{C}_{p}\right)$ than liquid nitrogen (typical freezing agent), allowing faster cooling rates. It is then expected that the CGW have a higher amount of HDL characteristic as its faster cooling rate generates amorphous ice with structure between liquid water and LDA (like a supercooled water) whereas HGW generates amorphous ice with more amount of LDL as its slower cooling rate gives enough time to generate LDL structures. It is important to mention that both CGW and HGW do not cross the $\tau_{c}$ avoiding crystal formation, therefore keeping the amorphous structure. 


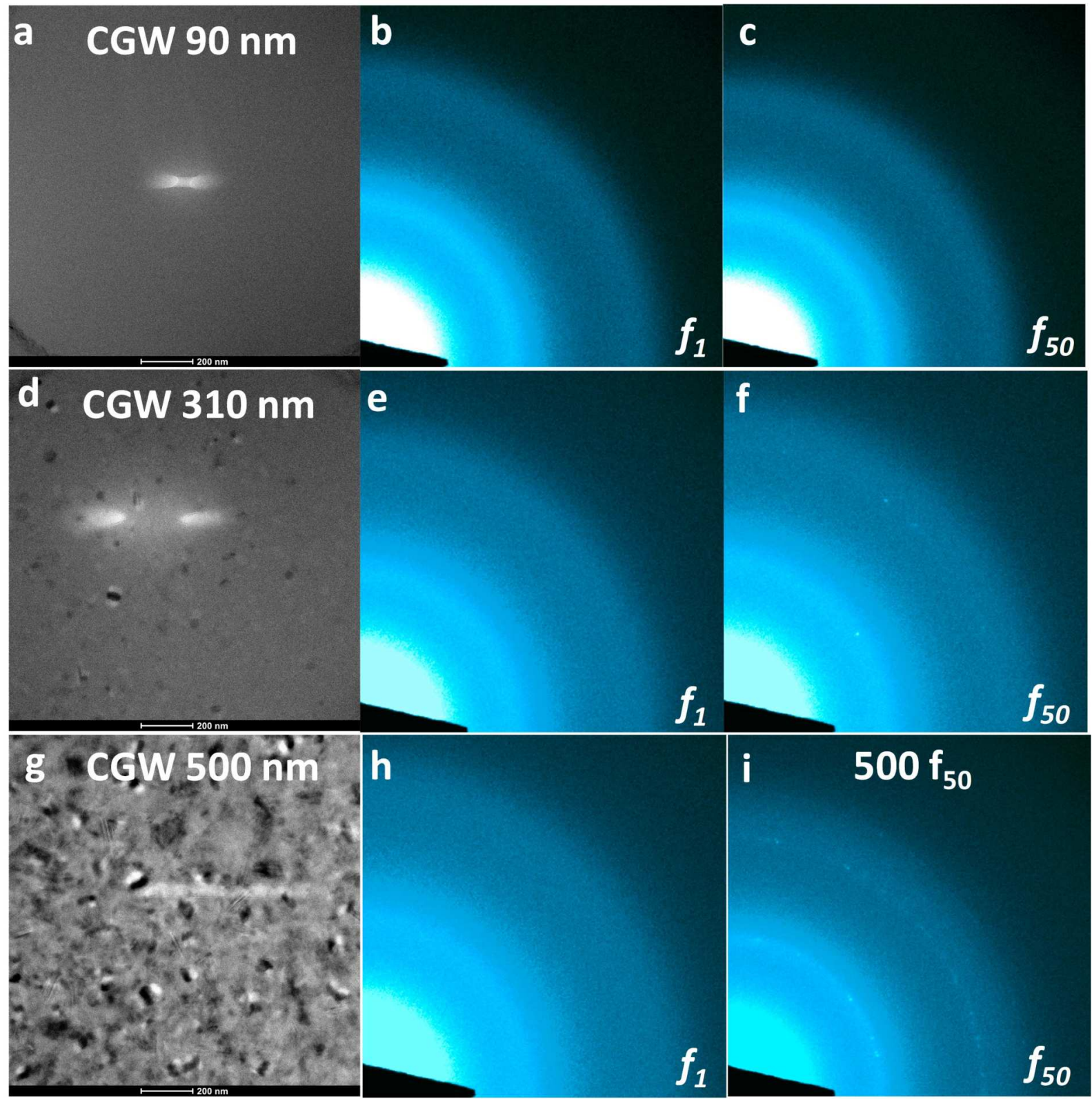

FIG. S14. Images and Electron Diffraction (ED) patterns of CGW films obtained with the TALOS F200C microscope $\left(Q_{\max }\right.$ of $\left.7.5 \AA^{-1}\right)$. For the 90 nm-thick sample: (a) its TEM image, (b) the first ED recorded at $0.3 \mathrm{~s}$, and (c) the last ED recorded at $15 \mathrm{~s}$. For the $310 \mathrm{~nm}$-thick sample: (d) TEM image, (e) first ED recorded at $0.3 \mathrm{~s}$, and (f) last ED recorded at 15s. Finally, for the 500 nm sample: (g) TEM image, (h) first ED at $0.3 \mathrm{~s}$, and (i) last ED at $15 \mathrm{~s}$. 


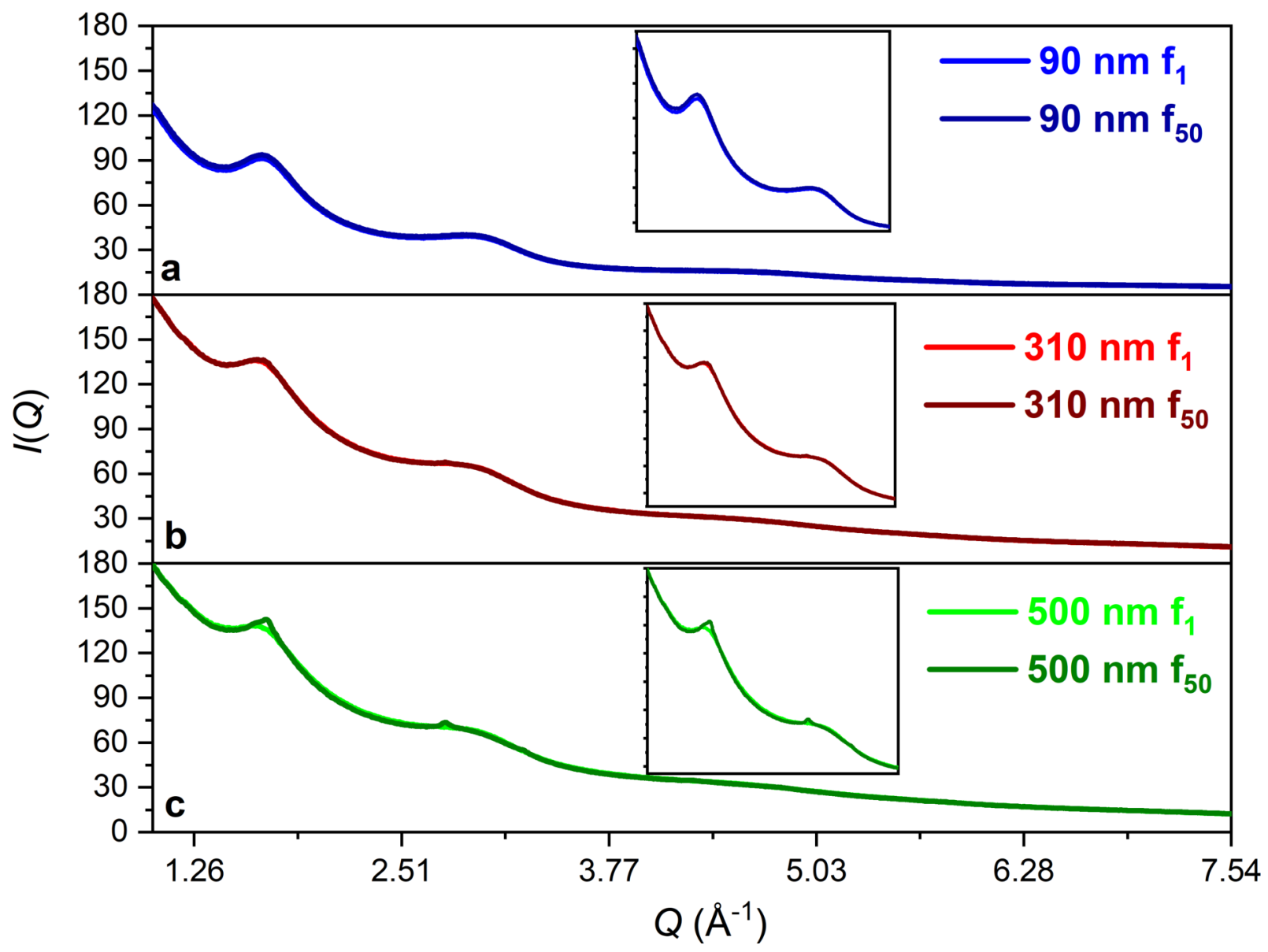

FIG. S15. Intensity scattering profiles $I(Q)$ for thin (a: $90 \mathrm{~nm})$, medium (b: $310 \mathrm{~nm})$, and thick (c: $500 \mathrm{~nm}$ ) Cryo-EM Glassy Water (CGW) films, respectively. Obtained with the TALOS F200C microscope $\left(Q_{\max }\right.$ of $\left.7.5 \AA^{-1}\right)$. In each case, frame 1 was recorded at $0.3 \mathrm{~s}\left(f_{1}\right)$ and frame 50 at 15 $\mathrm{s}\left(f_{50}\right)$ at an exposure rate of $\left(12 e^{-} / \AA^{2} \mathrm{~s}\right)$. These scattering profiles were obtained by rotational averaging the CGW selected-area electron-diffraction (SAED) patterns. 

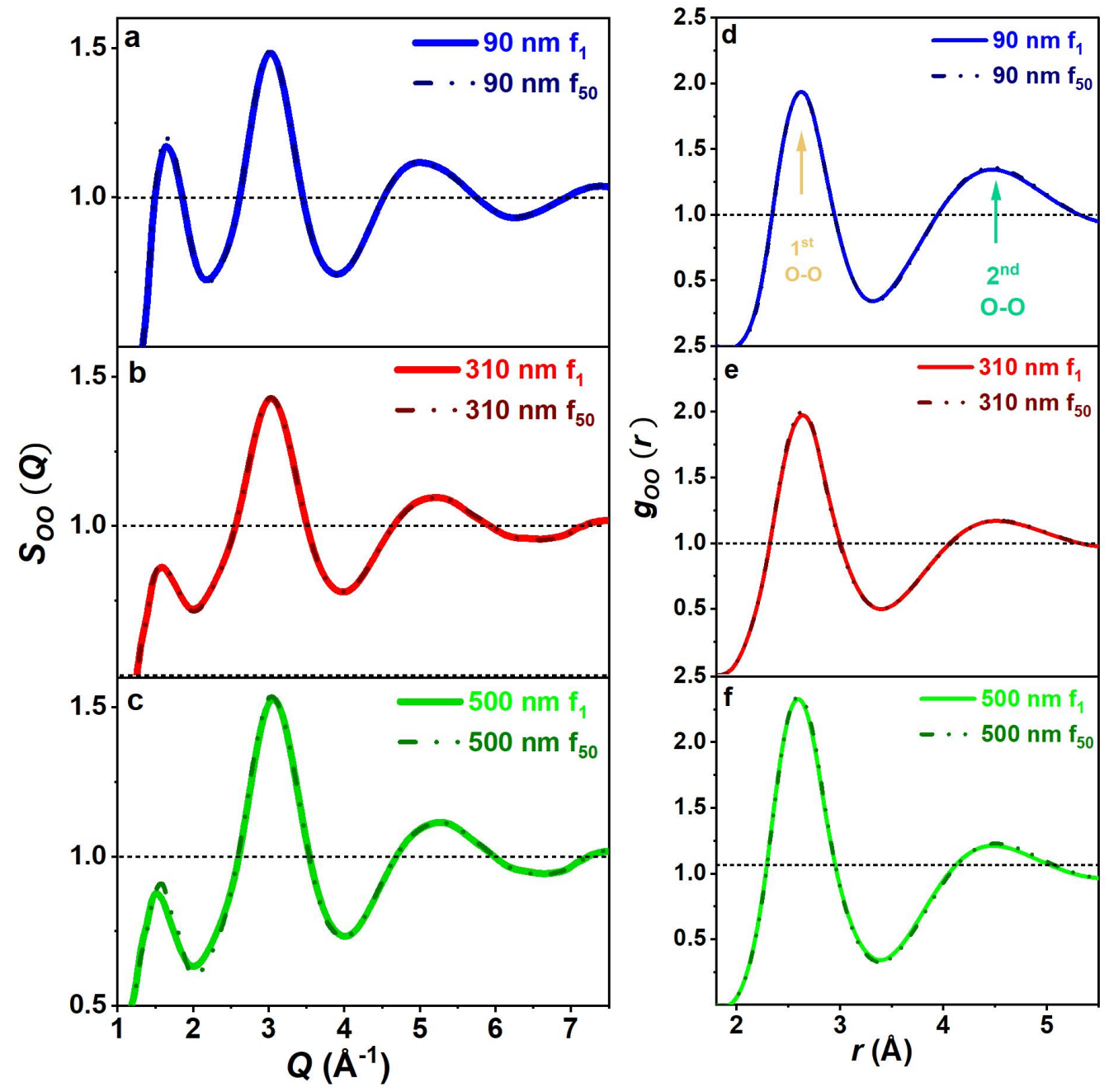

FIG. S16. Structure function $S_{O O}(Q)$ for: thin $(\mathbf{a}: 90 \mathrm{~nm})$, medium $(\mathbf{b}: 310 \mathrm{~nm})$, and thick (c: $500 \mathrm{~nm})$ Cryo-EM Glassy Water $(\mathrm{CGW})$ films, respectively. Frame 1 was recorded at $0.3 \mathrm{~s}\left(f_{1}\right)$ and frame 50 at $15 \mathrm{~s}\left(f_{50}\right)$ at an exposure rate of $\left(12 e^{-} / \AA^{2} \mathrm{~s}\right)$; Pair distribution functions (PDF) $g_{O O}(r)$ for the thin $(\mathbf{d})$, medium $(\mathbf{e})$, and thick $(\mathbf{f})$ samples. The low $g_{O O}(r)$ resolution is due to the $Q_{\max }$ used $(7.5 \AA)$, however it is observed that the electron exposure did not cause any structural change within the CGW matrix. Although we show in Figure 3 that ice $I_{c}$ nucleation occurs within the CGW matrix, the nucleation phenomenon is localized and does not cause changes on the remaining amorphous matrix structure. This is seen in Figure S14 where local ice crystals are observed and the amorphous content, Figure S15, has still the majority of the contribution to the scattering intensity profiles $I(Q)$. 


\section{REFERENCES}

[1] Shanmugam, J.; Borisenko, K. B.; Chou, Y. J.; Kirkland, A. I. eRDF Analyser: An Interactive GUI for Electron Reduced Density Function Analysis. SoftwareX 2017, 6, 185-192.

[2] Skinner, L. B.; Huang, C.; Schlesinger, D.; Pettersson, L. G.; Nilsson, A.; Benmore, C. J. Benchmark Oxygen-Oxygen Pair-Distribution Function of Ambient Water from X-Ray Diffraction Measurements with a Wide Q-Range. Journal of Chemical Physics 2013, 138.

[3] Mariedahl, D.; Perakis, F.; Späh, A.; Pathak, H.; Kim, K. H.; Camisasca, G.; Schlesinger, D.; Benmore, C.; Pettersson, L. G. M.; Nilsson, A.; Amann-Winkel, K. X-ray Scattering and O-O Pair-Distribution Functions of Amorphous Ices. Journal of Physical Chemistry B 2018, 122, $7616-7624$.

[4] Sorenson, J. M.; Hura, G.; Glaeser, R. M.; Head-Gordon, T. What Can X-Ray Scattering Tell Us About the Radial Distribution Functions of Water? The Journal of Chemical Physics 2000, 113, 9149-9161.

[5] Zeidler, A.; Salmon, P. S.; Fischer, H. E.; Neuefeind, J. C.; Simonson, J. M.; Markland, T. E. Isotope Effects in Water as Investigated by Neutron Diffraction and Path Integral Molecular Dynamics. J. Phys.: Condens. Matter 2012, 24, 284126.

[6] Zeidler, A.; Salmon, P. S.; Fischer, H. E.; Neuefeind, J. C.; Simonson, J. M.; Lemmel, H.; Rauch, H.; Markland, T. E. Oxygen as a Site Specific Probe of the Structure of Water and Oxide Materials. Phys. Rev. Lett. 2011, 107, 145501.

[7] Tran, D. T.; Svensson, G.; Tai, C.-W. SUePDF: A Program to Obtain Quantitative Pair Distribution Functions from Electron Diffraction Data. Journal of Applied Crystallography 2017, 50, 304-312.

[8] Stukowski, A. Visualization and Analysis of Atomistic Simulation Data with OVITO-the Open Visualization Tool. Modelling and Simulation in Materials Science and Engineering 2010, 18, 015012 .

[9] Van Der Spoel, D.; Lindahl, E.; Hess, B.; Groenhof, G.; Mark, A. E.; Berendsen, H. J. C. GROMACS: Fast, Flexible, and Free. Journal of Computational Chemistry 2005, 26, 17011718 . 
[10] Abraham, M. J.; Murtola, T.; Schulz, R.; Páll, S.; Smith, J. C.; Hess, B.; Lindahl, E. GROMACS: High Performance Molecular Simulations Through Multi-Level Parallelism from Laptops to Supercomputers. SoftwareX 2015, 1-2, 19-25.

[11] Abascal, J. L. F.; Sanz, E.; García Fernández, R.; Vega, C. A Potential Model for the Study of Ices and Amorphous Water: TIP4P/Ice. The Journal of Chemical Physics 2005, 122, 234511.

[12] Berendsen, H. J. C.; Postma, J. P. M.; van Gunsteren, W. F.; DiNola, A.; Haak, J. R. Molecular Dynamics with Coupling to an External Bath. The Journal of Chemical Physics 1984, 81, $3684-3690$.

[13] Darden, T.; York, D.; Pedersen, L. Particle Mesh Ewald: An N·log(N) Method for Ewald Sums in Large Systems. The Journal of Chemical Physics 1993, 98, 10089-10092.

[14] Essmann, U.; Perera, L.; Berkowitz, M. L.; Darden, T.; Lee, H.; Pedersen, L. G. A Smooth Particle Mesh Ewald Method. The Journal of Chemical Physics 1995, 103, 8577-8593.

[15] Allen, M. P.; Allen, M. P.; Tildesley, D. J.; ALLEN, T.; Tildesley, D. J. Computer Simulation of Liquids; Oxford Science Publ; Clarendon Press, 1989.

[16] Miyamoto, S.; Kollman, P. A. Settle: An Analytical Version of the SHAKE and RATTLE Algorithm for Rigid Water Models. Journal of Computational Chemistry 1992, 13, 952-962.

[17] Hess, B.; Bekker, H.; Berendsen, H. J. C.; Fraaije, J. G. E. M. LINCS: A Linear Constraint Solver for Molecular Simulations. Journal of Computational Chemistry 1997, 18, 1463-1472.

[18] Brehm, M.; Kirchner, B. TRAVIS - A Free Analyzer and Visualizer for Monte Carlo and Molecular Dynamics Trajectories. Journal of Chemical Information and Modeling 2011, 51, $2007-2023$.

[19] Seidl, M.; Fayter, A.; Stern, J. N.; Zifferer, G.; Loerting, T. Shrinking Water's no Man's Land by Lifting its Low-Temperature Boundary. Phys. Rev. B 2015, 91, 144201.

[20] Kim, K. H.; Späh, A.; Pathak, H.; Perakis, F.; Mariedahl, D.; Amann-Winkel, K.; Sellberg, J. A.; Lee, J. H.; Kim, S.; Park, J.; Nam, K. H.; Katayama, T.; Nilsson, A. Maxima in the Thermodynamic Response and Correlation Functions of Deeply Supercooled Water. Science 2017, 358, 1589-1593. 